\title{
Sporulation in Bacillus subtilis. Theoretical and Experimental Studies in Continuous Culture Systems
}

\author{
By I. W. DAWES AND J. H. M. THORNLEY* \\ Microbiology Unit, Department of Biochemistry, University of Oxford \\ South Parks Road, Oxford, OXI $3 Q U$
}

(Accepted for publication 17 March 1970)

\begin{abstract}
SUMMARY
A theoretical treatment of growth and sporulation of Bacillus subtilis Marburg strain I 68 in continuous culture is given. Sporulation is considered as a probability event, and a specific rate constant for its initiation in vegetative cells is introduced. The processes of growth and sporulation have been assumed to compete with each other in the vegetative organism. Equations are given which allow calculation of the rate constants characterizing growth and sporulation in steady-state continuous-flow systems. The relationship between the specific rate of initiation of sporulation and the growth rate of vegetative cells was determined with glucose-limited cultures and was approximately linear; the spore initiation rate increased with decreasing growth rate. This result was used to obtain equations relating the incidence of refractile spores to the dilution rate of a continuous culture maintained in a steady state. A number of possible models of the process of spore initiation have been considered; two were consistent with the linear relationship between spore initiation rate and growth rate determined by experiment.
\end{abstract}

\section{INTRODUCTION}

Continuous cultivation techniques have been applied by numerous workers to the study of spore or cyst formation by bacteria. Málek (1952) and Macura \& Kotková (1953) investigated the growth phases of Azotobacter in multistage continuous cultures; similar techniques were employed by Málek, Chaloupka, Vosyková \& Vinter (I953) to study sporulation in Bacillus pumilis. Sporulation of Bacillus cereus has been used by Ričica (1969) as an example of product formation in multistage continuous cultures . In single stage chemostats a number of attempts to study sporulation have been made, for example, in Bacillus megaterium (Aubert, Millet \& Castoriadis-May, 1961 ; Aubert, Millet \& Schaeffer, 1965); in Bacillus subtilis (Kerravala, Srinivasan \& Halvorson, 1964; Dawes \& Mandelstam, I969; Dawes, Kay \& Mandelstam, 1969); in Clostridium thermosaccharolyticum (Hsu \& Ordal, I969). Ierusalimskii (1958) and Ierusalimskii \& Rukina (1959) introduced a procedure whereby organisms growing in continuous culture could be viewed microscopically and thereby studied the reversibility of sporulation of clostridia and bacilli.

Despite this interest in spore formation in continuous culture, we are not aware of any attempt to develop a theoretical treatment of bacterial differentiation in continuous culture analogous to those carried out for vegetative bacterial growth, e.g.

\footnotetext{
* Present address: Glasshouse Crops Research Institute, Littlehampton, Sussex.
} 
Monod (I950), Novick \& Szilard (1950), Herbert, Elsworth \& Telling (I956), Herbert (I959, I96I), among others. This neglect may be due partly to the greater complexity of the system and partly to practical problems which have been encountered in continuous cultivation of spore-forming organisms. These include the selection of asporogenous mutants (Aubert et al. 196I), the failure of some spore-forming bacteria to attain a steady state (Kerravala, et al. 1964) or the germination of all but about I0 \% of the spores formed in single stage systems (E. O. Powell, personal communication).

This paper examines sporulation in continuous cultures in which growth is a function of the concentration of a single growth-limiting substrate; the possible dependence of sporulation on growth rate is investigated. The treatment is based on proposed models of sporulating systems; these models are evaluated in terms of experimental data obtained from steady state continuous culture systems.

\section{THEORY}

\section{General remarks concerning sporulation}

To describe formally a culture of bacteria undergoing spore formation it is first necessary to consider the nature of the sporulation process and to categorize the various populations of bacteria present in a culture undergoing both vegetative growth and sporulation.

Initiation of vegetative organisms to sporulation is thought to occur when certain nutrients necessary for growth become depleted (Büchner, I890; Knaysi, 1945; Grelet, I946, I952, 1957). However, Aubert et al. (196I) and Schaeffer, Millet \& Aubert (1965) showed that, in certain bacilli, sporulation occurred to some extent in minimal media, even when the bacteria were growing exponentially at their maximum rate. This observation led Schaeffer et al. (I965) to propose that initiation of a bacterium to spore formation could be considered as a probability event, an hypothesis confirmed by experiments with continuous culture in which Dawes \& Mandelstam (1969) found that bacteria were initiated to form spores with a probability dependent on the dilution rate.

Once initiated to spore formation the organisms pass through a sequence of morphological and biochemical changes including the production of protease, antibiotic, alkaline phosphatase, glucose dehydrogenase and dipicolinic acid and the appearance of refractility and heat resistance of the spores, etc. (Murrell, 1967; Mandelstam, I969).

Microbiologists, particularly those interested in continuous culture, describe cultures in terms of 'organism' as the basic unit; that is, in terms of 'recognizably separate entities' (Powell, 1956). For a sporulating system, however, there is no $a$ priori argument for adopting the organism level as the unit for quantitative representation of initiation to spore formation. In fact, members of the genus Bacillus can be found either as unicellular organisms, or as organisms containing more than one cell (up to multicellular filamentous forms). We have found that the majority of Bacillus subtilis (Marburg strain I68), under the conditions of continuous culture specified in this paper, are unicellular, some are bicellular and few occur as filamentous forms. Under favourable conditions every cell can form a spore (see Robinow, 1960) regardless of the number of cells in each organism. Under less favourable conditions 
cells within a filamentous organism may behave differently with respect to initiation of sporulation.

For these reasons the cultures have been described in terms of cell as the sporulating unit in this paper.

Cell populations present in sporulating systems

To analyse a sporulating bacterial culture, we assume it consists of three populations: vegetative cells (population $X$ ) which have not been initiated to form spores and are undergoing the normal process of cell division (all members of $X$ are assumed competent to form spores under appropriate conditions); population $Y$, which consists of cells that have become initiated to form spores, but which have not reached that stage of development in the sporulation sequence which is arbitrarily used as the criterion of spore formation (in this study the criterion is the appearance of refractility; once initiated, cells are presumed incapable of further division and do not contribute to increases in cell numbers); population $Z$, which have reached refractility or which have developed further (this class contains all free spores in addition to cells containing observable spores).

We use $n_{\mathrm{x}}, n_{\mathrm{y}}$ and $n_{\mathrm{z}}$ to denote the numbers per unit volume of culture of the cell types $X, Y$ and $Z$, respectively.

The spore initiation process

Little is known of the mechanism of initiation to sporulation of vegetative cells, particularly those in a growing culture. The theory developed in this paper is based on a treatment of spore initiation which resulted in the simplest mathematical description. Other feasible models (not given here) have been treated and lead to similar expressions.

Vegetative cells are treated as a single population which can undergo one of two competing processes: growth or sporulation

$$
\begin{aligned}
& X \stackrel{\nu}{\rightarrow} 2 X \quad \text { (growth), } \\
& X \stackrel{\kappa}{\rightarrow} Y \quad(\rightarrow Z) \quad \text { (sporulation), }
\end{aligned}
$$

in which $v$ and $\kappa$ are first-order rate constants. Once a cell has entered the spore formation sequence, we assume it always forms a mature spore at some later time and that there are no cells in the culture which are undergoing neither vegetative growth nor spore formation.

Next, the probability of initiation of a cell to sporulation before it divides is defined by $\phi$. As would be expected, the relationship of the probability $(\phi)$ to the rate constants ( $\nu$ and $\kappa$ ) depends upon the details of the particular treatment of the process of spore initiation chosen, although different models lead to similar expressions to that derived from the simple model outlined above, i.e.

$$
\phi=\frac{\kappa}{\nu+\kappa} .
$$

Kinetics of growth and initiation to sporulation

The growth of non-sporulating bacteria in terms of cell number $n_{\mathrm{x}}$ is usually described by the rate equation

$$
\frac{\mathrm{d} n_{\mathrm{x}}}{\mathrm{d} t}=\nu n_{\mathrm{x}},
$$

where $v$ is the specific number growth rate. 
Aubert et al. (196I) showed that bacteria formed spores during exponential growth, and that spores increased in proportion to the cell population. Thus a first order specific rate constant $\kappa$ for the process of initiation of vegetative cells to sporulation may be defined such that:

$$
\text { Rate of cell initiation to spore formation }=\kappa n_{\mathrm{x}} \text {. }
$$

Since we assume that growth and initiation are exclusive, the rate of increase of vegetative cells in a sporulating culture is given by

$$
\frac{\mathrm{d} n_{\mathrm{x}}}{\mathrm{d} t}=(\nu-\kappa) n_{\mathrm{x}} .
$$

Here $\nu$ is the specific growth rate of the cells if no sporulation were taking place. Increase in cell numbers is still exponential, but the effective growth rate is now $(\nu-\kappa)$.

\section{Kinetics of spore germination}

Most studies of the germination kinetics of spores have been carried out in cultures where an agent promoting germination has been applied suddenly (see Sussman \& Halvorson, I966). In such systems, Woese \& Morowitz (I958) found that germination can be approximately represented by first order kinetics. In the chemostat, however, spores are continually exposed to any germinant which may be present, and are heterogeneous with respect to germination rate (see experimental section).

For a system composed of $n$ spore fractions, each fraction $Z_{\mathrm{i}}$ germinating with first-order rate constant $\alpha_{i}$, and comprising a proportion $\lambda_{\mathbf{i}}$ of the total spore population, the rate equation for the change in total spore concentration, $n_{\mathrm{z}}$, at time $t$, due to germination is

$$
\frac{\mathrm{d}}{\mathrm{d} t}\left[n_{\mathrm{z}}(t)\right]=-\left[\sum_{i=1}^{n} \alpha_{\mathrm{i}}^{i} \lambda_{\mathrm{i}}(t)\right] n_{\mathrm{z}}(t)
$$

In a steady-state chemostat culture the proportion $\lambda_{i}$ will be constant at a given dilution rate and an overall first-order rate constant $\alpha$ can be used to describe germination, i.e.

$$
\alpha=\left[\sum_{i=1}^{n} \alpha_{\mathrm{i}} \lambda_{\mathrm{i}}\right] \text {. }
$$

The values of $\alpha$ vary to some extent with dilution rate since the $\lambda_{i}$ values vary with dilution rate.

Spore germination leads not only to a loss of spores from the culture, but contributes towards the vegetative cell population. There is a time lag between commencement of the germination process and outgrowth with the formation of a vegetative cell. This means that the rate of increase of vegetative cells at time $t$ is not given by $\alpha n_{\mathrm{z}}(t)$, but by $\alpha n_{\mathrm{z}}\left(t-t_{\mathrm{g}}\right)$ multiplied by a factor describing the loss of spores from the culture during the preceding time interval $t_{\mathrm{g}}$ (where $t_{\mathrm{g}}$ is the interval between the commencement of germination and cell outgrowth). For analysis of steady-state chemostat cultures it is reasonable to neglect this correction for washout since we found that at high dilution rates germination did not significantly contribute to any increase of vegetative cells, while at low dilution rates, where $\alpha n_{\mathrm{z}}$ is significant, the correction for $t_{\mathrm{g}}$ is not important. 
Growth and sporulation in the chemostat

The theoretical aspects of bacterial growth in single-stage homogeneous continuous cultivation systems given by Herbert et al. (I956) have been extended in the following treatment to the case of spore-forming bacteria.

Changes in the concentration of cell species. The completely mixed continuous culture system consists of a suitable vessel in which the culture is maintained at constant volume $v$. Sterile growth medium is fed into the vessel at constant flow rate $f$, and the vessel contents stirred so that there is uniform and rapid mixing. The dilution rate $D$ is defined by

$$
D=\frac{f}{v} .
$$

The time dependence of the vegetative cell (number) concentration, $n_{\mathrm{x}}$, is given by

$$
\frac{\mathrm{d} n_{\mathrm{x}}}{\mathrm{d} t}=\left(\nu-\kappa n_{\mathrm{x}}\right)-D n_{\mathrm{x}}+\alpha n_{\mathrm{z}} .
$$

The number of initiated cells at time $t, n_{\mathrm{y}}(t)$, is related to the values of $\kappa$ and $n_{\mathrm{x}}$ during the previous time interval $t-t_{0}$ to $t$, since there is a finite time interval between initiation of sporulation and appearance of a recognizably refractile spore. This time interval $t_{0}$ we call the sporulation time. At time $t-\tau$, the rate of initiation to sporulation from equation (3) is

$$
\kappa(t-\tau) n_{\mathrm{x}}(t-\tau)
$$

Of those cells initiated at time $t-\tau$, a fraction will wash out of the culture before reaching time $t$, i.e. before the cells reach an 'age from initiation' of $\tau$. This fraction of cells washed out is given by the exponential factor

$$
\exp \left(-\int_{t-\tau}^{t} D(\xi) \mathrm{d} \xi\right)
$$

Combining these last two expressions, at time $t$ the number of initiated cells with ages between $\tau$ and $\tau+\mathrm{d} \tau$ is

and $n_{\mathrm{y}}(t)$ is given by:

$$
\kappa(t-\tau) n_{\mathrm{x}}(t-\tau) \exp \left\{-\int_{t-\tau}^{t} D(\xi) \mathrm{d} \xi\right\} \cdot \mathrm{d} \tau
$$

$$
n_{\mathrm{y}}(t)=\int_{0}^{t_{0}} \kappa(t-\tau) n_{\mathrm{x}}(t-\tau) \exp \left\{-\int_{t-\tau}^{t} D(\xi) \mathrm{d} \xi\right\} \mathrm{d} \tau
$$

Finally, the equation governing $n_{z}$, the number concentration of entities recognizable as spores, is required. Cells are not recognized as containing spores until they have reached an age from initiation of $t_{0}$, hence the rate balance equation for $n_{\mathrm{z}}(t)$ is given by

$$
\frac{\mathrm{d} n_{\mathrm{z}}}{\mathrm{d} t}=\kappa\left(t-t_{0}\right) n_{\mathrm{x}}\left(t-t_{0}\right) \exp \left\{-\int_{t-t_{\mathrm{0}}} D(\xi) \mathrm{d} \xi\right\}-D n_{\mathrm{z}}-\alpha n_{\mathrm{z}}
$$

Changes in the concentration of substrate. Monod (1942) showed, for nonsporulating bacteria, the existence of an empirical relationship between the rate of bacterial growth and the rate of substrate utilization:

$$
\frac{\mathrm{d} n}{\mathrm{~d} t}=-y \frac{\mathrm{d} s}{\mathrm{~d} t}
$$


where $n$ is the concentration of bacteria and $y$ is known as the yield constant. This yield constant describes the rate of consumption of substrate by cells undergoing vegetative growth (Monod, 1950; Herbert et al. 1956).

In a culture undergoing sporulation as well as vegetative growth, growth-limiting substrate is undoubtedly consumed by cells which have been initiated to spore formation. However, the rate of consumption of substrate by initiated cells will depend on the age $\tau$ of the initiated cells. A function $\mathscr{C}(\tau)$ describing the rate of consumption of substrate by initiated cells $N_{\mathrm{y}}(t, \tau) \mathrm{d} \tau$ with ages between $\tau$ and $\tau+\mathrm{d} \tau$ can be defined by letting the rate of consumption of substrate be

$$
\mathscr{C}(\tau) N_{\mathrm{y}}(t, \tau) \mathrm{d} \tau
$$

From this, the total rate of change of substrate concentration by initiated cells is

$\frac{\mathrm{d} s}{\mathrm{~d} t}=D\left(s_{\mathrm{R}}-s\right)-\frac{1}{y} \cdot \frac{\mathrm{d} n_{\mathrm{x}}}{\mathrm{d} t}-\int_{0}^{t_{0}} \mathscr{C}(\tau) \kappa(t-\tau) n_{\mathrm{x}}(t-\tau) \exp \left\{-\int_{t-\tau}^{t} D(\xi) \mathrm{d} \xi\right\} \mathrm{d} \tau$.

The steady state

In the following equations the practice of Herbert et al. (1956), who use a tilde to denote steady-state values, will be followed.

When steady-state conditions obtain, $(\mathrm{d} / \mathrm{d} t)\left(n_{\mathrm{x}}\right),(\mathrm{d} / \mathrm{d} t)\left(n_{\mathrm{z}}\right)$ and $(\mathrm{d} s / \mathrm{d} t)$ are zero, and $\nu, \kappa$, and $D$ are time invariant, and equations (8), (9) and (I0) simplify respectively to

$$
\begin{aligned}
\nu-\kappa & =D-\alpha \frac{\tilde{n}_{\mathrm{z}}}{\tilde{n}_{\mathrm{x}}}, \\
\tilde{n}_{\mathrm{y}} & =\frac{\kappa \tilde{n}_{\mathrm{x}}}{D}\left[\mathrm{I}-\exp \left(-D t_{0}\right)\right], \\
\tilde{n}_{\mathrm{z}} & =\frac{\kappa \tilde{n}_{\mathrm{x}}}{D+\alpha} \exp \left(-D t_{0}\right) .
\end{aligned}
$$

The total cell concentration, $n_{\mathrm{T}}$, at steady state is given by:

$$
\tilde{n}_{\mathrm{T}}=\tilde{n}_{\mathrm{x}}+\tilde{n}_{\mathrm{y}}+\tilde{n}_{\mathrm{z}}=\tilde{n}_{\mathrm{x}}\left[\mathrm{I}+\frac{\kappa}{D}\left\{\left(\mathrm{I}-\exp \left(-D t_{0}\right)\right\}+\frac{\kappa}{D+\alpha} \exp \left(-D t_{0}\right)\right]\right.
$$

Finally, an equation relating substrate concentration to species concentrations can be obtained from equation (I3) if the function describing the rate of consumption of substrate by initiated cells is known. These aspects will not be developed further in this paper.

An observable characteristic of sporulating systems is the number of refractile spores as a fraction $(\theta)$ of the total population present in the culture, defined by

$$
\theta=\frac{n_{\mathrm{z}}}{n_{\mathrm{x}}+n_{\mathrm{y}}+n_{\mathrm{z}}} \text {. }
$$

From equations (I4), (15), (I6) and (I 8), the following relations can be derived;

$$
\begin{aligned}
& \tilde{\theta}=\frac{\kappa \exp \left(-D t_{0}\right)}{D+\kappa+\alpha\left[\mathrm{I}+\frac{\kappa}{D}\left\{\mathrm{I}-\exp \left(D t_{0}\right)\right\}\right]}, \\
& \kappa=\frac{D \tilde{\theta}(D+\alpha)}{D\left[\exp \left(-D t_{0}\right)-\tilde{\theta}\right]-\alpha \tilde{\theta}\left[\mathrm{I}-\exp \left(-D t_{0}\right)\right]}, \\
& \nu=D+\kappa\left\{\mathrm{I}-\frac{\alpha \exp \left(-D t_{0}\right)}{D+\alpha}\right\} .
\end{aligned}
$$


The probability of initiation of a vegetative cell to spore formation before it can undergo cell division has been assumed to be given by equation (I). Experimentally it is possible to measure $\tilde{\theta}, \alpha$ and $D$, and $t_{0}$ can be obtained by examining the transient variation of $\theta$ with time on making a shift in dilution rate, e.g. Dawes et al. (1969). Thus application of equations (20), (21) and (I) allows experimental evaluation of $\kappa, \nu$ and $\phi$, without the use of any assumptions concerning the detailed dependence of $\kappa$ and $\nu$ on the substrate concentration, $s$, provided $\tilde{\theta}$ values can be measured at particular dilution rates. However, in order to predict values for $\widetilde{\theta}, \kappa, \nu$, and $\phi$ given $D$ alone, it is necessary to determine the detailed dependence of $\kappa$ on $\nu$ or of $\kappa$ and $\nu$ on $s$. Moreover, these relationships must be available in order to begin to predict $n_{\mathrm{x}}, n_{\mathrm{y}}, n_{\mathrm{z}}, n_{\mathrm{T}}$ and $s$ as functions of $D$.

Variation of growth rate and spore initiation rate with substrate concentration. Some assumptions must now be made regarding factors affecting the specific rate constants $\nu$ and $\kappa$. We assume that both $\nu$ and $\kappa$ depend only on the concentration of the limiting substrate at time $t$, and are not affected by the prevailing levels of $n_{\mathrm{x}}, n_{\mathrm{y}}$ or $n_{\mathrm{z}}$, or by the previous history of the culture. We further assume that the specific rate constant for the increase of cell numbers, $\nu$ (which describes the rate of division of the bacteria and not the rate of increase of vegetative cell numbers in the sporulating population), varies with the limiting substrate concentration as

$$
\nu=\nu_{\mathrm{m}}\left(\frac{s}{K_{\mathrm{s}}+s}\right)
$$

as was first demonstrated by Monod (1942) for non-sporulating systems; $\nu_{\mathrm{m}}$ is the maximum specific growth rate of the bacteria at saturation levels of the limiting substrate, and $K_{\mathrm{s}}$ is a saturation constant.

It therefore remains to determine the variation of $\kappa$ (or $\phi$ ) with either $\nu$ or $s$. It has been shown that $\kappa$ and $\nu$ can be obtained experimentally by measuring $\theta$ values at various dilution rates. It is therefore possible to determine empirically the variation of $\kappa$ with $\nu$ and hence $\kappa$ with $s$ from equation (22).

\section{METHODS}

Organism. Bacillus subtilis (Marburg strain I68), auxotrophic for indole or tryptophan was stored as a spore suspension.

Continuous cultivation. The composition of the growth medium was as follows: $2 \cdot 78 \mathrm{~mm}$-glucose, $4.75 \mathrm{~mm}$-sodium citrate, 0.1 mm-tryptophan, $9.5 \mathrm{~mm}-\mathrm{NH}_{4} \mathrm{Cl}$, $20 \mathrm{~mm}^{-\mathrm{KH}_{2} \mathrm{PO}_{4} \text {, I mM-NaNO}}$, I mM-Na $_{2} \mathrm{SO}_{4}$, I mM-KCl, $2 \mathrm{~mm}-\mathrm{CaCl}_{2}, 0.0036 \mathrm{~mm}-$ $\mathrm{FeCl}_{3}, 0.04 \mathrm{I} \mathrm{mM-MgCl}, 0 . \mathrm{I} \mathrm{mM-MnCl}$; the $\mathrm{pH}$ was adjusted to 7.0 with $\mathrm{NaOH}$. Growth under these conditions was limited by the concentration of glucose. Cultivation was carried out at $37^{\circ}$ in a conical vessel of approximately $500 \mathrm{ml}$. capacity. The culture (about $100 \mathrm{ml}$.) was stirred by rapid aeration (I $1 . \mathrm{min}^{-1}$ of sterile-filtered humidified air). Problems of evaporation of the culture were avoided by saturation of the inlet air with water vapour. Sterile medium was fed into the vessel by means of a peristaltic pump. In order to maintain a near-continuous inflow of medium at low flow rates, an air-lock and a fine bore inlet were added to the feed line between the pumn and inlet.

Inoculation of the chemostat was carried out as described previously (Dawes at al. I969), and the culture allowed to reach equilibrium at various dilution rates. The 
attainment of a steady state was determined by observing the turbidity of the culture at $600 \mathrm{~nm}$. and by measuring the fraction of refractile spores in the culture.

Estimations. The incidence of refractile spores $(\theta)$ as a fraction of the total population was determined under the phase-contrast microscope, using cell samples taken from the chemostat and fixed immediately after sampling with formaldehyde. Samples were taken at intervals of about one generation time until there was no change in spore count; for these samples, sufficient cells were counted to include at least 100 spores: this corresponds to a $10 \%$ counting error.

The time taken for an initiated cell to form a refractile spore $\left(t_{0}\right)$ was determined by observing the effect of a sudden decrease in dilution rate of the chemostat system, as described previously (Dawes et al. 1969).

No suitable technique was available for estimation of the specific germination rate $(\alpha)$ of spores under chemostat conditions. However, germination rates were obtained for various spore fractions (resolved by equilibrium density gradient centrifugation of a sample from a steady-state culture) by suspending the spores in the supernatant of a steady-state chemostat culture and determining at intervals thereafter the fraction of spores which appeared phase-dark in the phase-contrast microscope. This supernatant was obtained by rapidly filtering $50 \mathrm{ml}$. of culture through a membrane filter (Millipore, $0.65 \mu$ pore size). From these data, plots of

$$
-\ln \frac{n_{\mathrm{z}}(t=t)}{n_{\mathrm{z}}(t=0)}
$$

against time $t$ were constructed for the various fractions and their germination rates determined from the slopes. The overall germination rate for the spore population was obtained from these rates and the proportions of spores present in each fraction using equation (6).

From equations (20), (2r) and (I) it can be seen that small errors in measurement of dilution rates could lead to much larger errors in estimation of the parameters, $\kappa, \nu$ and $\phi$. For this reason the flow rate of medium was obtained by measuring the time taken for $25 \mathrm{ml}$. of culture to flow from the chemostat. At least three series of measurements were taken at each steady state. The volume of culture in the chemostat vessel was measured directly after each experiment.

The maximum growth rate constant $\left(\nu_{\mathrm{m}}\right)$ was determined by observing the rate of increase of bacterial mass (estimated by turbidity measurements) from a low inoculum into the chemostat vessel containing fresh sterile medium. Rates were estimated at relatively low bacterial concentrations (less than $0.1 \mathrm{mg} . \mathrm{ml}^{-1}$ ), the average of three determinations was taken as $\nu_{\mathrm{m}}$.

Turbidity of the culture was measured at $600 \mathrm{~nm}$. using a SP 600 spectrophotometer. Measurements of $\mathrm{pH}$, using an EIL $2320 \mathrm{pH}$ meter, were taken as soon as possible after sampling from the culture.

Contaminants and asporogenous mutants were detected by plating samples of culture on various minimal-media plates. Bacillus subtilis 168 requires tryptophan for growth, hence contaminants were readily observed on tryptophanless minimalmedia plates. Asporogenous mutants were detected as white colonies on minimal-agar plates containing tryptophan: the parent organism formed brown colonies after several days incubation at $37^{\circ}$.

Three spore fractions were resolved from a steady-state culture by density-gradient 
centrifugation. An exponential gradient of Urografin was prepared over the density range $\mathrm{I} \cdot \mathrm{O}$ to $\mathrm{I} \cdot 25$, and the cells and spores obtained from a steady-state continuous culture sample ( $50 \mathrm{ml} . ; D=0.12 \mathrm{~h}^{-1}$ ) by centrifugation were suspended in I ml. of the culture supernatant and were layered onto the gradient. Fractions were then obtained by centrifugation at $45,000 \mathrm{~g}$ for $30 \mathrm{~min}$; ; one fraction pelleted due to its greater density $\left(>1.25 \mathrm{~g} . \mathrm{cm}^{-3}\right)$ and was found to contain free spores on microscopic examination. Two other fractions containing spores were obtained in the gradient and found to consist of spores included in lytic cells (mixed with germinating phasedark spores) and spores included in nonlytic cells.

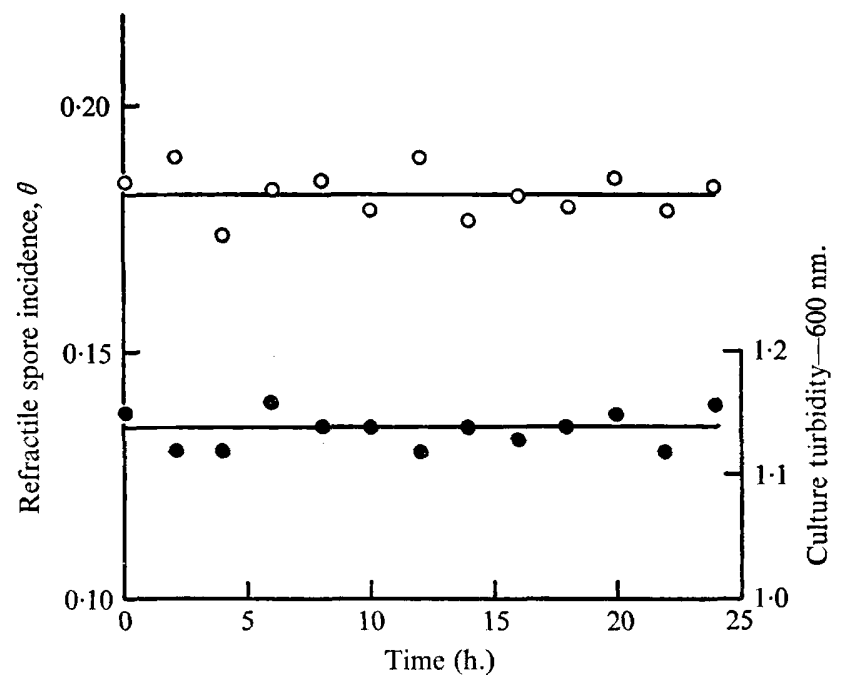

Fig. I. Sporulation in steady-state continuous culture of Bacillus subtilis 168 . A continuous culture of $B$. subtilis 168 was allowed to reach steady state under glucose limitation at a

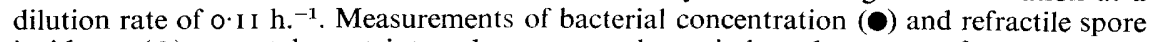
incidence $(O)$ were taken at intervals over a $24 \mathrm{~h}$. period, and were not found to differ significantly from straight lines of zero slope.

\section{RESULTS}

Stability of continuous cultures of Bacillus subtilis 168

Steady-state glucose-limited continuous cultures of Bacillus subtilis 168 , undergoing sporulation as well as vegetative growth, were obtained over the range of dilution rates from $0.05 \mathrm{~h}^{-1}$ to $0.39 \mathrm{~h} .{ }^{-1}$. These steady states were not maintained indefinitely with respect to spore formation, since asporogenous mutants appeared spontaneously and began to replace the sporulating organism to a significant extent after the steady state had been established for about 15 days.

Figure I gives an example of a steady-state culture (in which the dilution rate, $D$, was $\mathrm{O} \cdot \mathrm{I} \mathrm{I} \mathrm{h} .^{-1}$ ) before the observable appearance of mutants; measurements were taken over a $24 \mathrm{~h}$. period of the incidence of refractile spores $(\theta)$ and the turbidity of the culture. In general, therefore, experiments were not continued for longer than a week. 


\section{Parameters for treatment of data obtained from continuous cultures}

Germination rate $(\alpha)$. Spores formed in continuous cultures were found, by examination, to be heterogenous with respect to their germination characteristics. Three spore fractions of differing densities were obtained and germination rates for these fractions each suspended in filtrate from a steady-state culture were obtained, as described in Methods. The plots of

$$
-\ln \left[\frac{n_{\mathrm{z}}(t)}{n_{\mathrm{z}}(0)}\right] \text { versus } t
$$

are given in Fig. 2, the slopes of the straight lines in these plots were taken as estimates of the germination rates for the various spore fractions; these are given, together with the density range of the spores and the proportion of spores in each fraction, in Table $\mathrm{I}$.

Hence, as 'young spores' (those in nonlytic cells) germinate at faster rates than 'old spores', the specific germination rate for the population in the chemostat is not

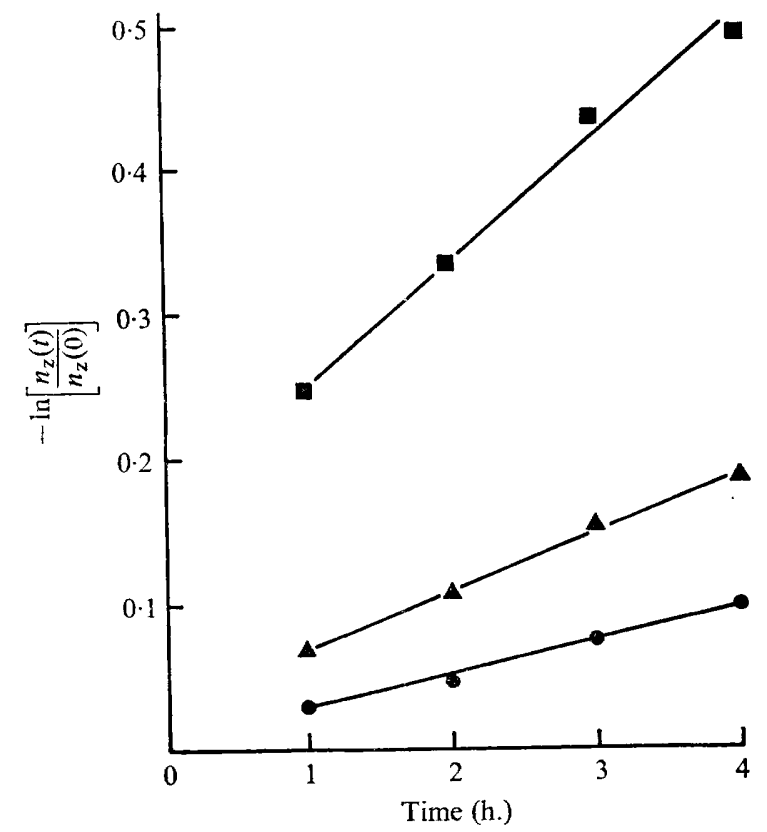

Fig. 2. Rates of germination of various spore fractions. Three spore fractions resolved from a chemostat spore population by equilibrium density-gradient centrifugation (see text) were each suspended in the rapid membrane filtrate of a steady-state culture and the refractile spore incidence determined at hourly intervals. The plots suggested in the text for estimating first-order germination rates for each of these fractions are given for: - spores from the most dense fraction; $\boldsymbol{\Delta}$ spores from the intermediate density fraction, and from the least dense fraction. $n_{z}(t)$ is the number of refractile spores per unit volume at time $t$.

constant for all dilution rates since it can be shown that the ratio of 'young spores' to 'old spores' varies with dilution rate. However, as was discussed in the theoretical section, the correction for germination is not important at higher dilution rates. Hence the value calculated for the above low dilution rate sample from the data in Table I and equation (6) of $\alpha=0.05 \mathrm{~h}^{-1}$ has been taken as a reasonable approximation for 
representation of the system. All data were treated using a range of $\alpha$ values in order to determine the effect of variation of this parameter on the interpretation of results.

The time for the formation of refractile spores $\left(t_{0}\right)$. The effect of imposing a sudden decrease in the dilution rate (from $0.4 \mathrm{~h}^{-1}$ to $0.05 \mathrm{~h}^{-1}$ ) on the incidence of refractile spores in a steady-state chemostat culture is shown in Fig. 3. The incidence of refractile

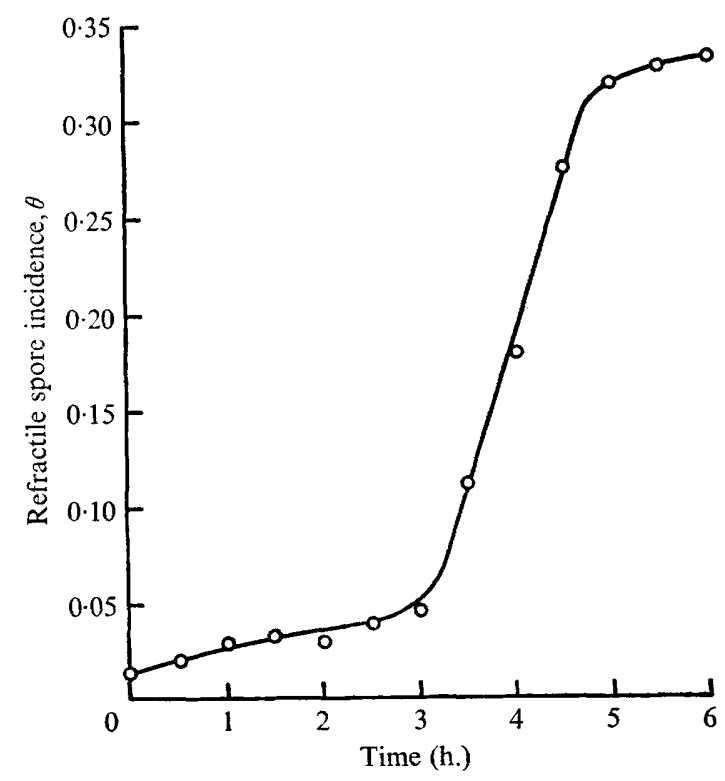

Fig. 3. The time for the formation of a refractile spore, $t_{0}$. At zero time the dilution rate of a steady-state culture at an initial value of $0.42 \mathrm{~h} .^{-1}$ was changed to $0.05 \mathrm{~h} .^{-1}$, and the incidence of refractile spores $(O)$ followed with time. At $3 \mathrm{~h}$. a sudden change in the spore incidence began which was complete at $5 \mathrm{~h}$. The average time for the change to occur $(4 \mathrm{~h}$.) has been taken as the time $\left(t_{0}\right)$ for the formation of a refractile spore.

Table I. The variation of germination rate of spore fractions from a continuous culture sample

Three fractions of the total spore population of a steady-state continuous culture sample ( $D=0.117 \mathrm{~h}^{-1}$ ) were obtained by equilibrium density gradient centrifugation. The first order germination rate for each fraction suspended in the filtrate of a steady state continuous culture $\left(D=0.123 \mathrm{~h}^{-1}\right)$ were obtained. Methods have been given in the text.

Type of spore

Spores included in non-lytic cells Spores included in lytic cells Spores free from parent cells

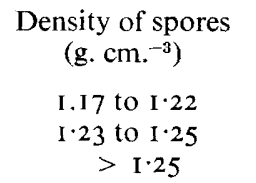

Proportion of spores

$\begin{array}{cc}\begin{array}{c}\text { Germination } \\ \text { rate }\left(\mathrm{h.}^{-1}\right)\end{array} & \begin{array}{r}\text { Proportion } \\ \text { of spores }\end{array} \\ 0.092 & 0.39 \\ 0.042 & 0.19 \\ 0.024 & 0.42\end{array}$

spores began to increase $3 \mathrm{~h}$. after the change, and reached a new steady-state value approximately $5 \mathrm{~h}$. after the change. The average time for the increase in refractile spores $\left(4^{\circ} \circ \mathrm{h}\right.$.) has been taken as the average time for formation of a refractile spore after initiation of a vegetative cell.

The maximum growth rate constant $\left(\nu_{\mathrm{m}}\right)$. For balanced growth under batch conditions, the mass growth rate and the number growth rate, $v$, must have the same value, 
Table 2. The effect of dilution rate on spore incidence in steady-state continuous culture of Bacillus subtilis I68

The continuous culture apparatus and experimental details are described in the text. Measurements were taken at each dilution rate until steady state was reached, and each row of figures refers to a different steady state. Six different chemostat runs were necessary to obtain this data, and the values obtained from each run are denoted by the superscripts $\mathrm{a}, \mathrm{b}, \ldots \mathrm{f}$.

\begin{tabular}{|c|c|c|c|}
\hline $\begin{array}{l}\text { Dilution rate } \\
\text { (D) } \mathrm{h}^{-1}\end{array}$ & $\begin{array}{l}\text { Refractile spore } \\
\text { fraction }(\theta)\end{array}$ & $\begin{array}{l}\mathrm{pH} \text { of culture } \\
\mathrm{pH} \text { units }\end{array}$ & $\begin{array}{c}\text { Turbidity of } \\
\text { culture } 600 \mathrm{~nm} \text {. }\end{array}$ \\
\hline $0.054^{\mathrm{c}}$ & 0.3060 & $7 \cdot 9$ & I. I 8 \\
\hline $0.055^{\mathrm{a}}$ & 0.3310 & $7 \cdot 9$ & 1.03 \\
\hline $0 \cdot 102^{\theta}$ & 0.1995 & $7 \cdot 7$ & $1 \cdot 00$ \\
\hline $0 \cdot \mathrm{III}^{\mathrm{b}}$ & 0.1820 & $7 \cdot 9$ & $I \cdot I 6$ \\
\hline $0.115^{b}$ & 0.1750 & $7 \cdot 9$ & $\mathrm{I} \cdot \mathrm{O} 4$ \\
\hline $0.116^{\mathrm{c}}$ & 0.1880 & - & - \\
\hline $0 \cdot 123^{\mathrm{d}}$ & $0 \cdot 1690$ & $7 \cdot 8$ & I.05 \\
\hline $0.132^{d}$ & 0.1219 & $7 \cdot 8$ & 1.02 \\
\hline $0.161^{a}$ & 0.1190 & $7 \cdot 9$ & 0.98 \\
\hline $0 \cdot 194^{\mathrm{e}}$ & 0.0750 & - & 0.94 \\
\hline $0.222^{b}$ & 0.0675 & $7 \cdot 5$ & 0.86 \\
\hline $0.257^{\mathrm{d}}$ & 0.0418 & $7 \cdot 5$ & 0.91 \\
\hline $0 \cdot 26 \mathrm{I}^{\mathrm{b}}$ & $0.050 \mathrm{I}$ & $7 \cdot 4$ & 0.86 \\
\hline $0.269^{d}$ & 0.0440 & $7 \cdot 2$ & 0.90 \\
\hline $0.280^{c}$ & 0.0347 & $7 \cdot 6$ & 0.94 \\
\hline $0 \cdot 299^{\mathrm{c}}$ & 0.0262 & $7 \cdot 7$ & 0.96 \\
\hline $0.303^{d}$ & 0.0295 & $7 \cdot 6$ & $0.9 \mathrm{I}$ \\
\hline $0 \cdot 307^{\mathrm{d}}$ & 0.0307 & $7 \cdot 6$ & 0.85 \\
\hline $0.380^{t}$ & 0.020 & $7 \cdot 4$ & 0.74 \\
\hline $0.387^{\mathrm{b}}$ & 0.013 & $7 \cdot 2$ & 0.68 \\
\hline
\end{tabular}

Table 3. Calculated data for steady-state cultures of Bacillus subtilis 168

Each row of figures refers to the values for the variables calculated from equations (I), (20) and $(21)$ and the data in Table 2 for each different steady state.

$\begin{array}{cccc}\begin{array}{c}\text { Dilution rate } \\ (D) \mathrm{h}^{-1}\end{array} & \begin{array}{c}\text { Specific growth } \\ \text { rate }(\nu) \mathrm{h}^{-1}\end{array} & \begin{array}{c}\text { Specific initiation } \\ \text { rate }(\kappa) \mathrm{h} .^{-1}\end{array} & \begin{array}{c}\text { Probability } \\ \text { initiation }\end{array} \\ 0.054 & 0.099 & 0.072 \mathrm{I} & 0.422 \\ 0.055 & 0.107 & 0.0844 & 0.440 \\ 0.102 & 0.156 & 0.0698 & 0.309 \\ 0.1 \mathrm{II} & 0.165 & 0.0679 & 0.29 \mathrm{I} \\ 0.1 \mathrm{II} & 0.169 & 0.0673 & 0.284 \\ 0.116 & 0.178 & 0.0762 & 0.300 \\ 0.123 & 0.18 \mathrm{I} & 0.0705 & 0.280 \\ 0.312 & 0.174 & 0.0496 & 0.222 \\ 0.16 \mathrm{I} & 0.217 & 0.0644 & 0.229 \\ 0.194 & 0.237 & 0.0477 & 0.167 \\ 0.222 & 0.272 & 0.0547 & 0.167 \\ 0.26 \mathrm{I} & 0.31 \mathrm{I} & 0.0527 & 0.145 \\ 0.257 & 0.296 & 0.0414 & 0.123 \\ 0.269 & 0.314 & 0.048 \mathrm{I} & 0.133 \\ 0.280 & 0.318 & 0.0399 & 0.1 \mathrm{I} \\ 0.299 & 0.33 \mathrm{I} & 0.0335 & 0.092 \\ 0.303 & 0.34 \mathrm{I} & 0.0393 & 0.103 \\ 0.307 & 0.347 & 0.0422 & 0.108 \\ 0.380 & 0.423 & 0.0437 & 0.094 \\ 0.387 & 0.415 & 0.0287 & 0.065\end{array}$


Hence the mean value (of three observations) obtained for the maximum mass growth rate as described in the Methods section $\left(0.487 \mathrm{~h}^{-1}\right)$ was taken as the maximum growth rate constant, $\nu_{\mathrm{m}}$.

\section{Quantitative data for steady-state cultures at various dilution rates}

The steady-state data obtained from six separate runs in the same chemostat are summarized in Table 2, and the calculated values of $\kappa, \nu$ and $\phi$ for each steady-state sample are given in Table 3 and are plotted against the dilution rate $(D)$ in Fig. 6, 7 and 8. Fig. 5 gives the values of $\theta$ against $D$. For calculation, the values $\alpha=0.05 \mathrm{~h}^{-1}$ and $t_{0}=4^{\circ} \mathrm{o} \mathrm{h}$. were used. A plot of $\kappa$ versus $\nu$ is given in Fig. 4. This was found to be approximately linear.

Regression analysis gave the relationship

$$
\kappa=0.09 \mathrm{I}-0 \cdot 143^{\nu}
$$

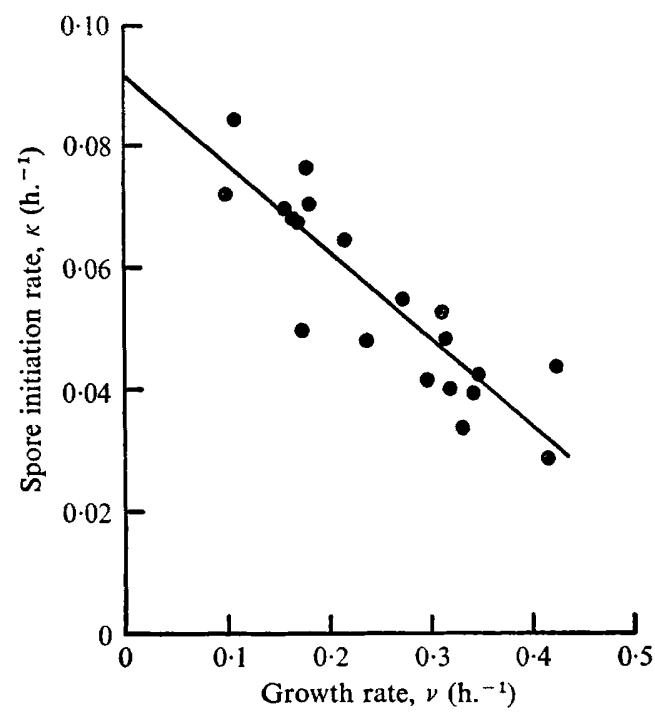

Fig. 4

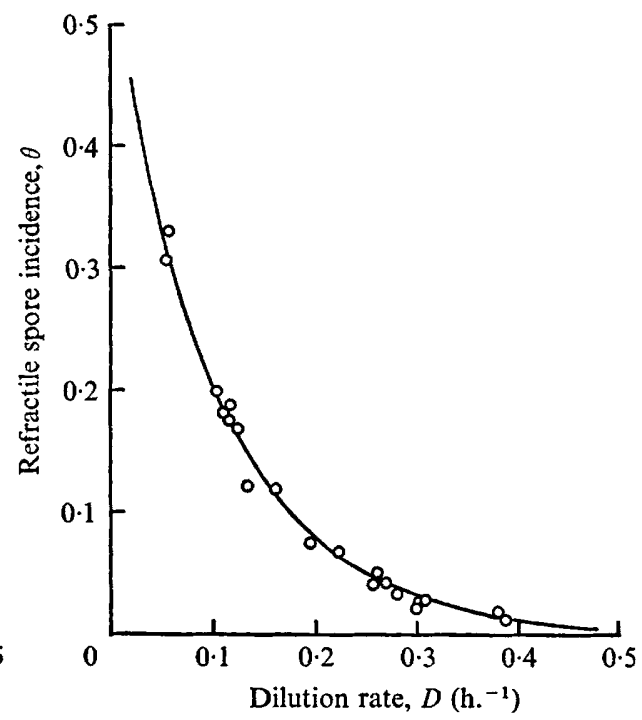

Fig. 5

Fig. 4. Variation of the rate of sporulation initiation with growth rate of Bacillus subtilis 168 in steady-state continuous culture. Values for the rate of sporulation initiation $(\kappa)$ and the growth rate $(v)$ obtained experimentally from glucose-limited continuous cultures at different steady-state dilution rates were plotted against each other. The continuous line is that obtained from a linear regression analysis of $\kappa$ on $\nu$, and is represented by equation (23).

Fig. 5. The relationship between the refractile spore incidence at steady state in a continuous culture and dilution rate. Data for the refractile spore incidence given in Table 2 are plotted against dilution rate for comparison with the theoretical relationship predicted from equations (19), (21) and (23). Open circles represent experimental points, each point represents the refractile spore incidence in the culture after a particular steady state had been established. The continuous line represents the theoretical curve.

\section{Test of the theoretical treatment}

In addition to the calculation of $\kappa, v$ and $\phi$ values using the observed values for $\alpha$ and $t_{0}$, various combinations of $\alpha$ values (from o h..$^{-1}$ to $0^{\cdot} \mathrm{I} \mathrm{h} .^{-1}$ ) and $t_{0}$ values (from $3.0 \mathrm{~h}$. to $5^{\circ} \mathrm{Oh}$.) were used in treatment of the data in Table 2 in order to evaluate the 
effect of errors in measurement of these parameters on the interpretation. If an $\alpha$ value of $0.005 \mathrm{~h}^{-1}$ or less is used in the calculation of $\kappa$ and $\nu$, the resulting values of $\kappa$ are almost constant over the range of dilution rates employed. This situation is, however, unlikely since germinating spores have been observed in steady-state cultures by phasecontrast or electron microscopy in numbers which indicate that the $\alpha$ value must be close to that used to calculate the data in Table 3, i.e. $\alpha=0.05 \mathrm{~h}^{-1}$.

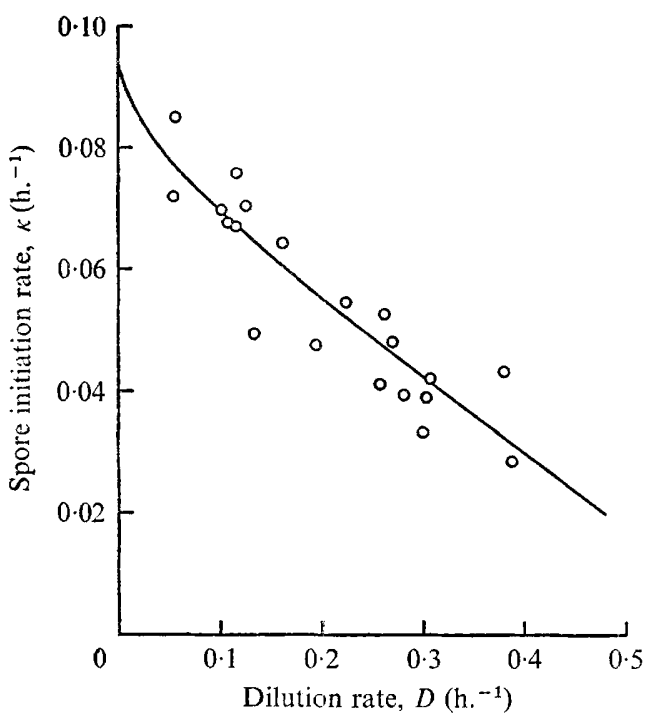

Fig. 6

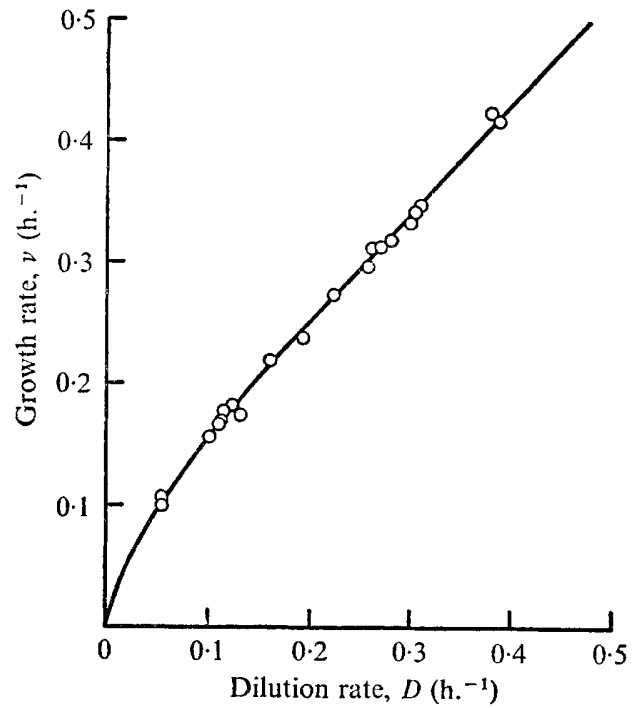

Fig. 7

Fig. 6. The quantitative relationship of the rate of sporulation initiation to dilution rate of a steady-state continuous culture of Bacillus subtilis $\mathbf{I} 68$. Values for the sporulation initiation rate $(\kappa)$ calculated from the experimental data as described in the text are plotted against dilution rate $(D)$. The open circles correspond to measurements taken on a culture after a particular steady state had been established (see Table 3). For comparison, the theoretical curve obtained from equations (2I) and (23) is given as the continuous line.

Fig. 7. The quantitative relationship of the growth rate to the dilution rate of a steady-state continuous culture of Bacillus subtilis $\mathrm{I} 68$. Values for the growth rate $(v)$ calculated from the experimental data as described in the text are plotted against the dilution rate $(D)$. Each open circle corresponds to a particular steady state, and the theoretical curve obtained from equations (21) and (23) is given as the continuous line.

Prediction of $\theta, \kappa, \nu$ and $\phi$ values. With knowledge of the two constants arising from the linear relationship between $\kappa$ and $\nu$, it is possible to predict $\tilde{\theta}, \kappa, \nu$ and $\phi$ values, given the dilution rate $D$, without knowing the values for either the yield constants for substrate utilization or the saturation constant for growth rate $\left(K_{\mathrm{s}}\right)$ using equations $(21)$ (23), (19) and (I). By substitution of equation (23) in equation (2I), $\nu$ can be obtained as a function of $D$ and the constants $\alpha$ and $t_{0}$, and from this the variation of $\kappa, \tilde{\theta}$ and $\phi$ follow directly from the above equations.

Theoretical curves for $\tilde{\theta}, \kappa, \nu$ and $\phi$ against $D$ have been obtained from $\alpha=0.05 \mathrm{~h}^{-1}$, $t_{0}=4.0 \mathrm{~h} ., \nu_{\mathrm{m}}=0.487 \mathrm{~h}^{-1}$, and using equation (23). These have been plotted in Fig. 5 to 8 together with the experimental data. It can be seen that good quantitative agreement exists between the theoretical predictions and the experimental values. 


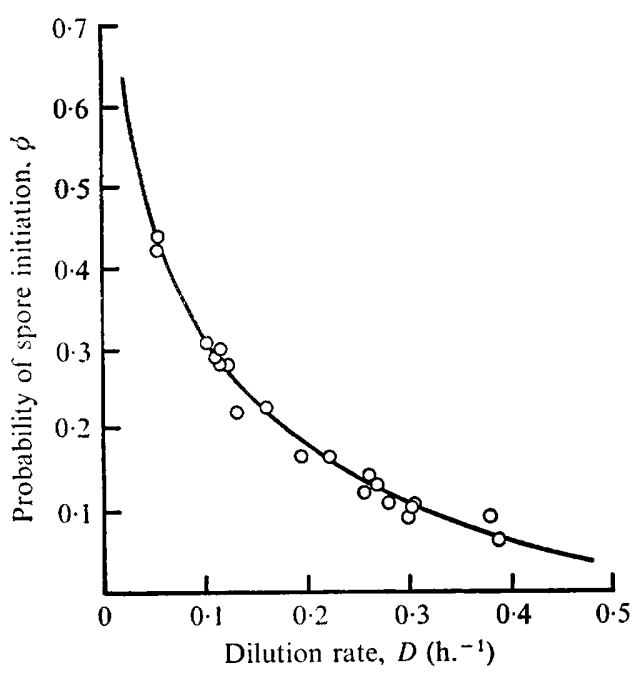

Fig. 8. The quantitative relationship of the probability of initiation of a vegetative cell to sporulation to the dilution rate of a steady-state continuous culture of Bacillus subtilis I68. Values for the probability of spore initiation $(\phi)$ calculated from the data of Table 2 as described in the text are plotted against the dilution rate $(D)$. Open circles correspond to particular steady states, and the continuous line represents the theoretical curve obtained from equations (1), (2I) and (23).

\section{DISCUSSION}

In the sporulating system, it is clear that a single yield constant will not, in principle, be sufficient to describe the substrate utilization of the very heterogeneous population. A theoretical basis for a description of substrate utilization in a sporulating culture has been briefly discussed, although in the absence of good data concerning substrate levels prevailing in the culture vessel, these aspects of the problem were not pursued further. The concentration of limiting substrate was not measured due to the difficulty of obtaining accurate data at low dilution rates; such a difficulty was encountered in experiments with glycerol limitation in chemostat cultures of Aerobacter cloacae (Herbert et al. 1956). We must state at this point that this lack of data about substrate concentrations led us to assume (without direct experimental verification) that Monod's relation, equation (22), describes the dependence of growth rate $(\nu)$ on the substrate concentration $(s)$. This assumption does not affect the conclusions drawn concerning the linear dependence of the specific rate of spore initiation on growth rate, nor is it implicit in the treatment of the second model of spore initiation which appears in the following discussion. Nor is this assumption required for prediction of the observed variation of $\kappa, \nu, \theta$ and $\phi$ with dilution rate. The assumption has been made in the first model of spore initiation.

A new and important parameter in the present treatment is the specific rate constant $\kappa$, which describes the process of initiation of vegetative cells to spore formation. Assumptions which simplify the relation of $\kappa$ to growth and probability of sporulation were chosen in order to keep the mathematics simple, and those assumptions leading to more complicated mathematics added nothing. 
With all these assumptions (all of which can be criticized) we have shown that for Bacillus subtilis 168 cultures under the conditions specified here, the relationship between the spore initiation rate $\kappa$ and the cell division rate $\nu$ is approximately linear and is of the form of equation (23)

$$
\kappa=a-b v
$$

where $a=0.09 \mathrm{I} \mathrm{h}^{-1}$ and $b=0.143$.

\section{Models of the spore initiation process}

The empirical relationship found between the spore initiation rate and the growth rate provides a method for testing models of the spore initiation process. We have found two very different models of spore initiation to predict the observed behaviour and these are set out briefly below.

Model I. Repression model. There have been several suggestions made in the literature that sporulation in bacilli is controlled by the level of intracellular metabolite(s), see Schaeffer et al. (I965), Mandelstam (1969), Hsu \& Ordal (I969). Schaeffer et al. (1965) drew an analogy between sporulation control and catabolite repression. The following repression system is analogous to the mechanism of control of the synthesis of an inducible enzyme postulated by Jacob \& Monod (196I). Its postulates are:

(i) Spore formation is initiated by a repression system in which an 'effector' metabolite $(E)$ reacts wtih a target, $T_{1}$ (say a protein)

$$
E+T_{1} \rightleftharpoons T_{1} E
$$

and the molecule $T_{1} E$ reacts with a second target $T_{2}$ (say a site on the chromosome)

$$
T_{1} E+T_{2} \rightleftharpoons T_{1} T_{2} E \text {. }
$$

(ii) Both reactions are reversible and in equilibrium.

(iii) The concentration of the effect or metabolite is a function of the limiting substrate concentration, $s$. The concentrations of the 'targets' ([ $\left.T_{1}\right]$ and $\left.\left[T_{2}\right]\right)$ do not vary with $s$.

(iv) The probability of initiation of a cell to spore formation is proportional to the fraction of $T_{2}$ target sites unoccupied at any particular instant, i.e.

$$
\phi=\frac{\left[T_{2}\right]}{\left[T_{2}\right]+\left[T_{1} T_{2} E\right]} .
$$

(v) The concentration of effector metabolite $[E]$ is proportional to the limiting substrate concentration, $s$.

By straightforward application of these postulates the probability $\phi$ can be shown o vary with substrate concentration as

$$
\phi=\frac{a+b}{a+b s},
$$

where $a, b$ are constants derived from the concentrations of the targets and the equilibrium constants for the reactions postulated in (i) and $b=\mathrm{I}$.

From this, by means of equations (I) and (22), the linear relationship, equation (23), of $\kappa$ to $\nu$ can be derived.

Model 2. Initiation occurs at constant frequency in cells containing nonreplicating chromosomes. This model was suggested by Professor J. Mandelstam (personal communication) as an interesting alternative to repression models. Its postulates are:

(i) Cells can be initiated to spore formation only when they contain nonreplicating 
chromosomes. (This assumption leaves open the question of what control mechanism prevents expression of the spore genes when DNA-replication is occurring.)

(ii) The 'time gap' between successive rounds of DNA replication increases linearly with generation time for generation times in excess of a minimum DNA-replication time $\tau_{\text {rep }}$. Initiation occurs at constant rate in cells containing nonreplicating chromosomes.

Using these postulates it can be shown that

$$
\kappa=\gamma\left(1-\mathrm{I} \cdot 44 \tau_{\text {rep }} \nu\right),
$$

i.e. a linear relationship of the form (equation (23)):

$$
\kappa=a-b v \text {. }
$$

If this model were correct, the constants $(a$ and $b$ ) determined empirically would enable one to estimate the minimum DNA-replication time $\tau_{\text {rep }}$. From the data in the experimental section $\tau_{\text {rep }}$ would have a value of $\mathrm{I} \cdot 09 \mathrm{~h}$. (cf. $0.7 \mathrm{~h}$. for Escherichia coli found by Lark, I966, and Helmstetter \& Cooper, I968).

Neither of the above models may in fact represent the actual process of spore initiation. At the moment, evidence obtained from other studies tends to support hypotheses based on the involvement of 'inhibition' of sporulation by small molecule metabolites. For example, growth limitation of bacilli in continuous culture by means of an auxotrophic requirement or an essential ion leads to much lower incidences of spores than that found under conditions of carbon or nitrogen limitation (Aubert et al. 1965; Dawes \& Mandelstam, 1969). However, it is possible that 'time gaps' between DNA replications vary differently with growth rate when different substrates limit growth. No conclusive evidence in support of either hypothesis (or any other) is currently available. The results presented here do, however, provide a method for testing the validity of hypotheses concerning the control of sporulation initiation.

The authors thank Professor J. Mandelstam for helpful discussion, criticism and advice. One of us (J.H.M.T.) is indebted to St Catherine's College and the Ford Foundation for a Research Fellowship. I.W.D. gratefully acknowledges the receipt of a Rhodes Scholarship.

\section{REFERENCES}

Aubert, J. P., Millet, J. \& Castoriadis-May, C. (I96I). Relation entre le taux de croissance et la sporulation chez Bacillus megaterium. Compte Rendu Hebdomadaire des Séances de l'Académie. des Sciences, Paris 253, 173 I-1733.

Aubert, J. P., Millet, J. \& Schafffer, P. (1965). Croissance et sporulation de Bacillus megaterium en culture continue. Compte Rendu Hebdomadaire des Séances de l'Académie des Sciences, Paris 26r, 2407-2413.

BUCHNER, H. (I 890). Ueber die Ursache der Sporenbildung beim Milzbrandbacillus. Zentralblatt für Bakteriologie, Parasitenkunde, Infektionskrankheiten und Hygiene (Abteilung I) 8, I-6.

Dawes, I. W. \& Mandelstam, J. (1969). Biochemistry of sporulation of Bacillus subtilis i 68 : continuous culture studies. In Continuous Cultivation of Micro-organisms. Proceedings of the 4th Symposium, Prague, 1968, pp. I57-162. Prague: Academia, Publishing House of the Czechoslovak Academy of Sciences.

Dawes, I. W., Kay, D. \& Mandelstam, J. (1969). Sporulation in Bacillus subtilis. Establishment of a time scale for the morphological events. Journal of General Microbiology 56, I7I-I79.

Grelet, N. (1946). Sporulation d'une souche de Bacillus megatherium par épuisement soit du fer, soit du carbone, soit de l'azote en milieu synthétique. Compte Rendu Hebdomadaire des Séances de l'Academié des Sciences, Paris 222, 4I8-420. 
Grelet, N. (1952). La detérminisme de la sporulation de Bacillus megatherium, IV. Constituants minéraux de milieu synthétique nécessaires à la sporulation. Annales de l'Institut Pasteur, Paris 83, 7I-79.

Grelet, N. (1957). Growth limitation and sporulation. Journal of Applied Bacteriology 20, 315-324.

Helmstetter, C. E. \& COOPER, S. (I968). DNA synthesis during the division cycle of rapidly growing Escherichia coli B/r. Journal of Molecular Biology 3I, 507-5I 8 .

Herbert, D. (1959). Some principles of continuous culture. In Recent Progress in Microbiology, pp, 38I-396. Stockholm: Almquist Wiksell.

Herbert, D. (1961). A theoretical analysis of continuous culture systems. In Continuous Culture of Micro-organisms. Monograph no. 12, pp. 2I-53. London: Society of Chemical Industry.

Herbert, D., Elsworth, R. \& Telling, R. C. (1956). The continuous culture of bacteria. A theoretical and experimental study. Journal of General Microbiology 14, 60I-622.

Hsu, E. J. \& Ordal, Z. J. (I969). Sporulation of Clostridium thermosaccharolyticum under conditions of restricted growth. Journal of Bacteriology 97, I 5 I I-I 5 I 2.

IERUSALIMSKII, N. D. (1958). A study of the process of development of micro-organisms by the continuous flow and exchange of media method. Continuous Cultivation of Micro-organisms. A Symposium, pp. 62-66. Prague: Academia, Publishing House of the Czechoslovak Academy of Sciences.

IerusalimskiI, N. D. \& Rukina, N. A. (1959). Studies on the conditions of spore formation by the method of continuous microcultures. Mikrobiologiya, Moscow 28, 80 I-806.

JACOB, F. \& MONOD, J. (196I). Genetic regulatory mechanisms in the synthesis of proteins. Journal of Molecular Biology 3, 31 8-356.

Kerravala, D. J., Srinivasan, V. R. \& Halvorson, H. O. (I964). Endogenous factor in sporogenesis in bacteria. II. Growth and sporulation in Bacillus subtilis. Journal of Bacteriology 88, 374-380.

KNAYSI, G. (1945). A study of some environmental factors which control endospore formation by a strain of Bacillus mycoides. Journal of Bacteriology 49, 473-493.

LARK, K. G. (1966). Regulation of chromosome regulation and segregation in bacteria. Bacteriological Reviews 30, 3-32.

Macura, J. \& Kotková, M. (I953). Development of Azotobacter in continuous medium. Continuous cultivation of micro-organisms. Ceskoslovenská Biologie, Prague 2, 49-58 (in Russian).

MÁlek, I. (1952). Kultivierung von Azotobakter in strömendem Milieu. Českoslolovenská Biologie, Prague r, 39-47 (in Russian).

Málek, I., Chaloupka, J., Vosyková, L. \& Vinter, V. (I953). Sporulation of bacilli. Československá Biologie, Prague 2, 323-337 (in Russian).

Mandelstam, J. (1969). Regulation of bacterial spore formation. Symposia of the Society for General Microbiology 19 377-402.

Monod, J. (1942). Recherches sur la croissance des cultures bactériennes. Paris: Hermann et Cie.

Monod, J. (1950). La technique de culture continue. Théorie et application. Annales de l'Institut Pasteur, Paris 79, 390-410.

Murrell, W. G. (1967). The biochemistry of the bacterial endospore. In Advances in Microbial Physiology I, 133-25I.

Novick, A. \& Szilard, L. (I950). Description of the chemostat. Science, New York Ir2, 71 5-7I6.

POWELL, E. O. (I956). Growth rate and generation time of bacteria, with special reference to continuous culture. Journal of General Microbiology 15, 492-5 II.

ŘIČICA, J. ( I969). Sporulation of Bacillus cereus in multistage continuous cultivation. In Continuous Cultivation of Micro-organisms. Proceedings of the 5th Symposium, pp. 163-I7I. Prague: Academia, Publishing House of the Czechoslovak Academy of Sciences.

RoBINow, C. F. (1960). Morphology of bacterial spores; their development and germination. In The Bacteria, vol. I, pp. 207-248. Edited by I. C. Gunsalas \& R. Y. Stanier. London \& New York: Academic Press.

Schaeffer, P., Millet, J. \& Aubert, J. P. (I965). Catabolite repression of bacterial sporulation. Proceedings of the National Academy of Sciences of the United States of America 54, 704-71 I.

Sussman, A. S. \& Halvorson, H. O. (1966). Spores, Their Dormancy and Germination, pp. I28-139. New York \& London: Harper \& Row.

WoESE, C. \& MOROwITZ, H. J. (1958). Kinetics of the release of dipicolinic acid from spores of Bacillus subtilis. Journal of Bacteriology 76, 8I-83. 